\title{
Clinical phenotypes and outcomes of precapillary pulmonary hypertension of sickle cell disease
}

\author{
Laurent Savale (1) ${ }^{1,2,3}$, Anoosha Habibi ${ }^{4,5}$, François Lionnet ${ }^{6}$, Bernard Maitre (1) 7 , \\ Vincent Cottin $\mathbb{1}^{8}$, Xavier Jais ${ }^{1,2,3}$, Ari Chaouat $\mathbb{1}^{9,10}$, Elise Artaud-Macari ${ }^{11}$, \\ Matthieu Canuet (10 ${ }^{12}$, Grégoire Prevot ${ }^{13}$, Christelle Chantalat-Auger ${ }^{14}$, \\ David Montani (10 ${ }^{1,2,3}$ Olivier Sitbon (10 ${ }^{1,2,3}$. Fréderic Galacteros ${ }^{4,5}$ \\ Gérald Simonneau ${ }^{1,2,3}$, Florence Parent ${ }^{1,2,3}$, Pablo Bartolucci (10 ${ }^{4,5}$ and \\ Marc Humbert (10 ${ }^{1,2,3}$
}

@ERSpublications

Clinical phenotype of precapillary pulmonary hypertension of sickle cell disease is influenced by the genotype. Thrombotic lesions appear as a major component of $\mathrm{PH}$ related to $\mathrm{SCD}$, more frequently in SC patients. http://bit.ly/32b2nEx

Cite this article as: Savale L, Habibi A, Lionnet F, et al. Clinical phenotypes and outcomes of precapillary pulmonary hypertension of sickle cell disease. Eur Respir J 2019; 54: 1900585 [https://doi.org/10.1183/ 13993003.00585-2019].

\section{ABSTRACT}

Rationale: Precapillary pulmonary hypertension $(\mathrm{PH})$ is a devastating complication of sickle cell disease (SCD). Little is known about the influence of the SCD genotype on PH characteristics.

Objectives: To describe clinical phenotypes and outcomes of precapillary PH due to SCD according to disease genotype.

Methods: A nationwide multicentre retrospective study including all patients with SCD-related precapillary $\mathrm{PH}$ from the French $\mathrm{PH}$ Registry was conducted. Clinical characteristics and outcomes according to SCD genotype were analysed.

Results: 58 consecutive SCD patients with precapillary PH were identified, of whom 41 had homozygous for haemoglobin S (SS) SCD, three had S- $\beta_{0}$ thalassaemia (S- $\beta_{0}$ thal) and 14 had haemoglobin SC disease (SC). Compared to SC patients, $S S / S-\beta_{0}$ thal patients were characterised by lower 6 -min walk distance $(p=0.01)$ and lower pulmonary vascular resistance $(p=0.04)$. Mismatched segmental perfusion defects on lung scintigraphy were detected in $85 \%$ of SC patients and $9 \%$ of SS/S- $\beta_{0}$ thal patients, respectively, and $50 \%$ of $S S / S-\beta_{0}$ thal patients had heterogeneous lung perfusion without segmental defects. After $\mathrm{PH}$ diagnosis, 31 patients (53\%) received medical therapies approved for pulmonary arterial hypertension, and chronic red blood cell exchange was initiated in 23 patients (40\%). Four patients were managed for chronic thromboembolic $\mathrm{PH}$ by pulmonary endarterectomy $(\mathrm{n}=1)$ or balloon pulmonary angioplasty $(\mathrm{n}=3)$. Overall survival was $91 \%, 80 \%$ and $60 \%$ at 1,3 and 5 years, respectively, without influence of genotype on prognosis.

Conclusions: Patients with precapillary PH related to SCD have a poor prognosis. Thrombotic lesions appear as a major component of $\mathrm{PH}$ related to SCD, more frequently in SC patients.

This article has been revised according to the correction published in the January 2020 issue of the European Respiratory Journal.

This article has supplementary material available from erj.ersjournals.com

Received: 25 March 2019 | Accepted after revision: 28 Aug 2019

Copyright @ERS 2019 


\section{Introduction}

Pulmonary hypertension $(\mathrm{PH})$ has been identified as a devastating complication of sickle cell disease (SCD) leading to a higher risk of mortality [1]. Screening studies combining transthoracic echocardiography and right heart catheterisation (RHC) for definite diagnosis have reported a $\mathrm{PH}$ prevalence ranging from 6 to $10 \%$ in SCD [2-4]. Among these patients, less than half had precapillary PH defined by mean pulmonary arterial pressure (mPAP) $\geqslant 25 \mathrm{mmHg}$ and pulmonary artery wedge pressure (PAWP) $\leqslant 15 \mathrm{mmHg}$. Histological studies from SCD patients demonstrate pulmonary vascular remodelling and thrombotic lesions which may result in a progressive elevation of pulmonary vascular resistance (PVR) and ultimately right heart failure $[4,5]$. Additional factors like shear stress, hyperviscosity or comorbidities (such as portal hypertension, chronic thromboembolic disease, interstitial lung disease, left cardiac dysfunction) could also contribute to the pulmonary haemodynamic derangement [6]. The complex and multifactorial physiological mechanisms of precapillary $\mathrm{PH}$ in this specific setting leads to a wide range of phenotypes and haemodynamic profiles. The effect of chronic haemolysis on nitric oxide (NO) depletion and endothelial dysfunction, combined with thrombotic lesions, seem to be the two main mechanisms responsible for chronic pulmonary vascular disease in SCD. Regardless of underlying mechanisms, it has been clearly established that $\mathrm{PH}$ contributes to impaired functional status in patients with SCD. Severe exertional dyspnoea and reduced exercise capacity, as measured by the 6-min walk distance (6MWD), has been observed in all clinical studies of SCD patients with PH. Furthermore, the prognosis of SCD patients with precapillary $\mathrm{PH}$ is severely compromised $[2,4,7]$.

Despite the functional and prognostic implications of $\mathrm{PH}$ in SCD, specific guidelines on the management of SCD-associated precapillary PH are scarce due to a paucity of evidence [8].

All main genotypes of SCD seem to be associated with $\mathrm{PH}$, and we hypothesised that some differences in the clinical and haemodynamic characteristics may exist that could influence outcomes and management. While haemolysis seems to be closely linked to the severity of $\mathrm{PH}$ in some patients, others are developing genuine chronic thromboembolic PH (CTEPH), justifying specific management [9-13]. A more precise description of phenotypes observed in all subpopulations of SCD patients with $\mathrm{PH}$ could support better personalised management. The aim of this study was to describe and compare the clinical characteristics and outcomes of all consecutives cases of precapillary PH related to SCD of the French PH Registry according to SCD genotype (homozygous for haemoglobin S (SS) SCD or S- $\beta_{0}$ thalassaemia (S- $\beta_{0}$ thal) versus haemoglobin SC disease).

\section{Methods}

Study population

Data from all consecutive SCD-associated precapillary $\mathrm{PH}$ patients referred to the French National Reference Centre for PH (Université Paris-Sud, Le Kremlin-Bicêtre) or expert centres from the French PH Network between January 2000 and December 2017 were collected from the French PH Registry.

SCD was confirmed by haemoglobin electrophoresis. All patients were screened for $\mathrm{PH}$ with transthoracic Doppler echocardiography and were subsequently referred to a $\mathrm{PH}$ expert centre if $\mathrm{PH}$ was suspected. Precapillary $\mathrm{PH}$ was defined by a mPAP $\geqslant 25 \mathrm{mmHg}$ and a PAWP $\leqslant 15 \mathrm{mmHg}$ measured by RHC [14, 15]. Cases of postcapillary $\mathrm{PH}$ defined by $\mathrm{mPAP} \geqslant 25 \mathrm{mmHg}$ and PAWP $>15 \mathrm{mmHg}$ were excluded. Haemodynamic assessment was systematically performed at least 1 month after an acute vaso-occlusive crisis. In line with current guidelines, all patients with a diagnosis of precapillary PH confirmed by RHC underwent extensive investigations in order to identify possible causes or risk factors for pulmonary

Affiliations: 'Univ. Paris-Sud, Faculté de Médecine, Université Paris-Saclay, Le Kremlin-Bicêtre, France. ${ }^{2}$ AP-HP, Service de Pneumologie, Centre de Référence de l'Hypertension Pulmonaire, Hôpital Bicêtre, Le Kremlin-Bicêtre, France. ${ }^{3}$ INSERM UMR_S 999, Hôpital Marie Lannelongue, Le Plessis Robinson, France. ${ }^{4}$ APHP, Hôpitaux Universitaire Henri Mondor, Unité des Maladies Génétiques du Globule Rouge, Centre de Référence des Pathologies du Globule Rouge, Créteil, France. ${ }^{5}$ UPEC, Institut Mondor de Recherche Biomédicale (IMRB), Institut National de la Santé et de le Recherche Médicale (INSERM) U955, DHU A-TVB, Créteil, France. ${ }^{6}$ AP-HP, Service de Médecine Interne, Hôpital Tenon, Paris, France. ${ }^{7}$ AP-HP, Hôpitaux Universitaires Henri Mondor, Unité de Pneumologie, Créteil, France. ${ }^{8}$ Université Claude-Bernard Lyon 1, Hôpital Louis-Pradel, Service de Pneumologie, Centre de Référence National des Maladies Pulmonaires Rares, UMR154, Lyon, France. 'Département de Pneumologie, CHRU de Nancy, rue du Morvan, Vandœuvrelès-Nancy, France. ${ }^{10}$ INSERM U1116, Université de Lorraine, Nancy, France. ${ }^{11}$ Pneumology Dept, Rouen University Hospital, Rouen, France. ${ }^{12}$ Service de Pneumologie, Nouvel Hôpital Civil, Hôpitaux Universitaires de Strasbourg, Strasbourg, France. ${ }^{13}$ Service de Pneumologie, Hôpital Larrey, Toulouse, France. ${ }^{14}$ AP-HP, Service de Médecine Interne, Hôpital Bicêtre, Le Kremlin-Bicêtre, France.

Correspondence: Laurent Savale, Université Paris-Sud, Centre de Référence de l'Hypertension Pulmonaire Service de Pneumologie et Soins Intensifs Respiratoires, Hôpital Bicêtre, 78, rue du Général Leclerc, 94270 Le Kremlin-Bicêtre, France. E-mail: laurent.savaledaphp.fr 
arterial hypertension (PAH), such as congenital heart disease, portal hypertension, HIV infection or connective tissue disease $[14,15]$. Moreover, the presence of chronic lung disease and CTEPH was assessed by performing pulmonary function tests, high-resolution computed tomography of the chest, ventilation/perfusion (V/Q) lung scintigraphy followed by computed tomography pulmonary angiography (CTPA) and/or digital subtraction pulmonary angiography (DSA) if needed. Chronic restrictive lung disease was defined as a total lung capacity of $<70 \%$ associated with a chest radiographic pattern of interstitial lung disease. Baseline evaluation included physical examination, assessment of modified New York Heart Association functional class (NYHA-FC), routine blood tests, non-encouraged 6MWD, pulmonary function tests and arterial blood gases. Characteristics of patients at baseline were compared according to the disease genotype.

The study complied with the Declaration of Helsinki. Although French law does not require ethics committee approval or informed consent for retrospective data collection, the data collected were anonymised and complied with the requirements of the Commission Nationale de l'Informatique et des Libertés, the organisation dedicated to privacy, information technology and civil rights in France. The committee approved the methods used to collect and analyse data on 24 May 2003 (approval number 842063).

\section{Multidisciplinary management}

All patients received supportive therapies in accordance with $\mathrm{PH}$ guidelines, i.e. diuretics to control signs and symptoms of right ventricular failure (including peripheral oedema) and long-term oxygen therapy if hypoxaemia was present. Initiation of long-term anticoagulation therapy was left to the discretion of physicians according to the assessment of the possible contribution from in situ thrombosis and/or thromboembolic disease to $\mathrm{PH}$.

Off-label use of medical therapies approved for $\mathrm{PAH}$, i.e. endothelin-receptor antagonists (ERA), phosphodiesterase type-5 (PDE-5) inhibitors or prostanoids, was decided after a multidisciplinary discussion of the expected benefits and risks of this medical strategy and was systematically recorded. CTEPH patients necessitating specific management with medical therapies, balloon pulmonary angioplasty or pulmonary endarterectomy were discussed in a multidisciplinary meeting including experienced surgeons for pulmonary endarterectomy, interventional radiologists/cardiologists, radiologists experienced in pulmonary vascular imaging and pulmonologists with expertise in $\mathrm{PH}$, as recommended in the guidelines $[14,15]$.

Decisions to use hydroxyurea and/or initiation of chronic red blood exchange transfusion after precapillary $\mathrm{PH}$ diagnosis were also systematically validated by a multidisciplinary meeting and recorded.

\section{Statistical analysis}

Continuous variables are expressed as median (range) values. Differences in continuous variables, such as patients' age, $6 \mathrm{MWD}$ and haemodynamic characteristics, were compared using the Mann-Whitney U-test. Categorical variables were expressed as number and percentage and were compared using the chi-square test for independence. Baseline and post-baseline values for haemodynamic variables were compared using a Wilcoxon matched-pairs signed rank test. A p-value of $<0.05$ was considered statistically significant.

The Kaplan-Meier method was used to estimate survival at each time point. For the overall survival analysis after diagnosis, we used the first haemodynamic evaluation as the starting point to determine the survival duration. The cut-off date was 30 June 2018. Patients who were lost to follow-up were censored at the date of the last available visit.

Univariate analysis based on the Cox proportional hazards model was used to examine the relationship between survival and selected baseline variables. Results are expressed as hazard ratios with $95 \%$ confidence intervals.

\section{Results}

Patient demographics and clinical characteristics at the time of precapillary PH diagnosis

58 consecutive SCD patients with precapillary PH were identified, including 41 with SS, 3 with S- $\beta_{0}$ thal and 14 with SC disease. A comparison of clinical characteristics at baseline between SC and SS/S- $\beta_{0}$ thal patients is reported in table 1 . A predominance of female patients in the SS/S- $\beta_{0}$ thal group and a predominance of male patients in the SC group was observed. As expected, the haemoglobin level was significantly higher and biomarkers of haemolysis (lactate dehydrogenase and bilirubin) were significantly lower in the SC group compared with the $S S / S-\beta_{0}$ thal group. Pulmonary function tests showed a significantly lower forced vital capacity $(\mathrm{FVC})$ in the $S S / S-\beta_{0}$ thal group whereas diffusing capacity for carbon monoxide adjusted for haemoglobin and arterial blood gases $\left(P_{\mathrm{aO} 2}\right.$ and $\left.P_{\mathrm{aCO}_{2}}\right)$ were similar in both 
groups. Distribution of NYHA-FC at time of diagnosis was similar between both groups of patients. In contrast, $6 \mathrm{MWD}$ was significantly lower in SS/S- $\beta_{0}$ thal patients. Finally, RHC showed a lower cardiac index and higher PVR in SC patients. The distribution of PVR according to genotype is illustrated in figure 1. A majority of SS/S- $\beta_{0}$ thal patients (54\%) had PVR lower than $3 \mathrm{WU}$ and a small proportion of patients had high levels of PVR (three patients presented with PVR $>10 \mathrm{WU}$ ). In SC patients PVR values were more homogeneously distributed with a median of $5.3 \mathrm{WU}$.

11 patients presented with significant chronic restrictive lung disease. Two patients had portal hypertension, one connective tissue disease (antisynthetase syndrome with interstitial lung disease) and one mediastinal sarcoidosis.

TABLE 1 Characteristics at time of $\mathrm{PH}$ diagnosis according to the disease genotype

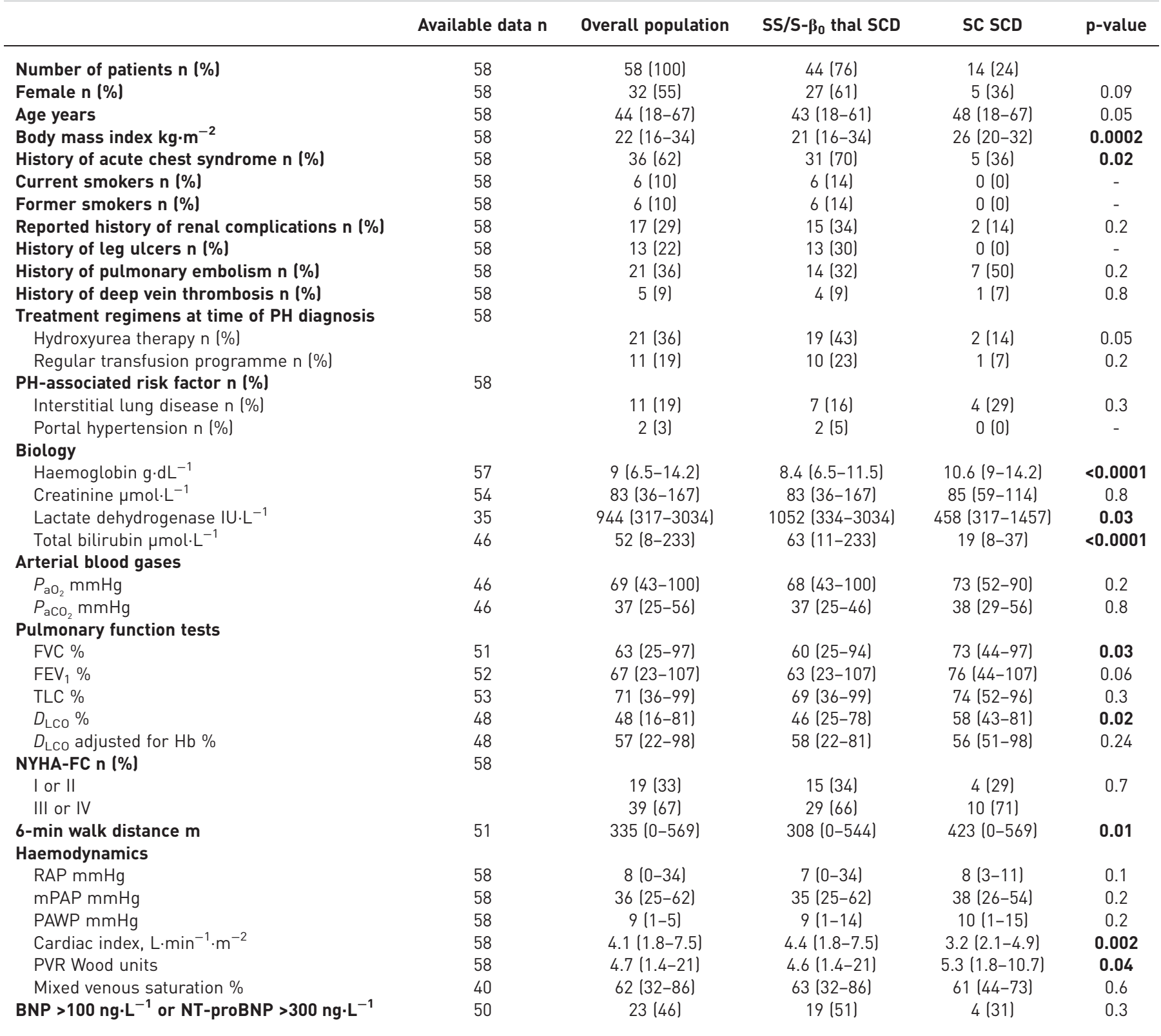

BNP: brain natriuretic peptide; $D_{\mathrm{LCO}}$ : diffusing capacity for carbon monoxide; $\mathrm{FEV}_{1}$ : forced expiratory volume in $1 \mathrm{~s}$; FVC: forced vital capacity; mPAP: mean pulmonary arterial pressure; NT-proBNP: N-terminal pro-brain natriuretic peptide; $P_{\mathrm{aO}_{2}}$ : arterial oxygen tension; $P_{\mathrm{aco}}$ : arterial carbon dioxide tension; NYHA-FC: New York Heart Association functional class; PAWP: pulmonary arterial wedge pressure; PH: pulmonary hypertension; PVR: pulmonary vascular resistance; RAP: right atrial pressure; TLC: total lung capacity. 
a)

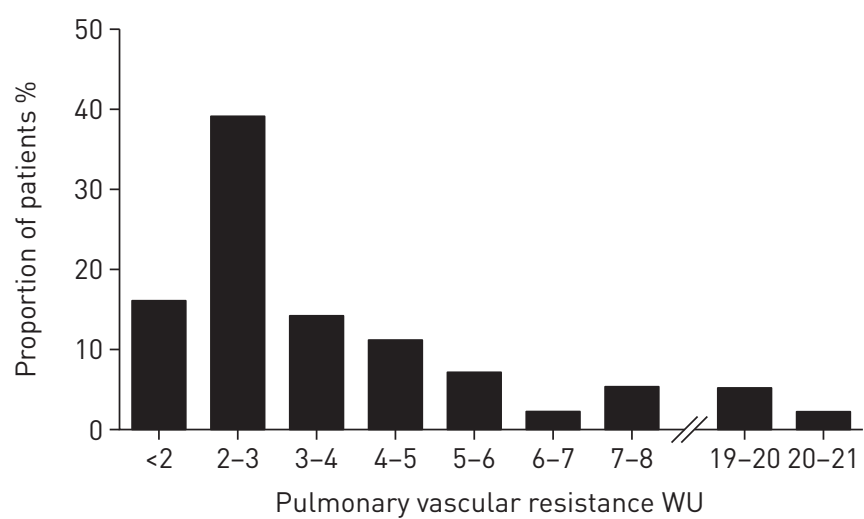

b)

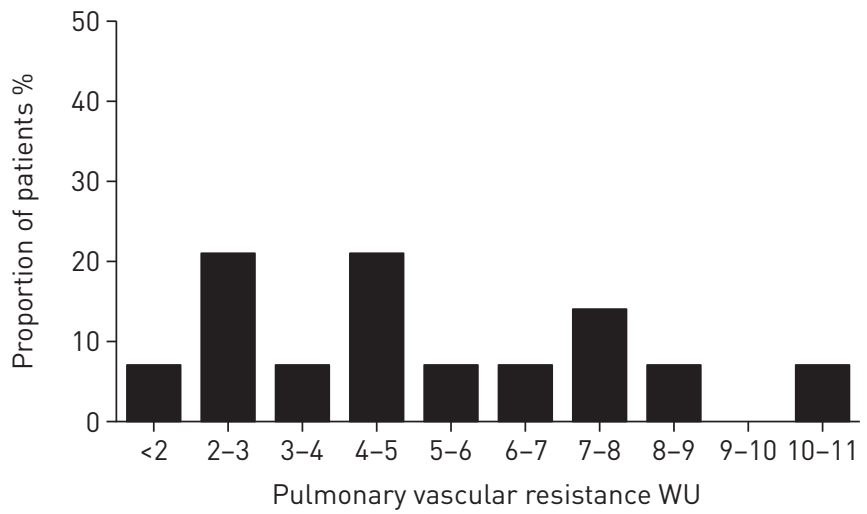

FIGURE 1 Pulmonary vascular resistance distribution according to sickle cell disease genotype.

A history of acute venous thromboembolism was reported in 7 out of 14 SC patients (50\%) and 13 out of $44 \mathrm{SS} / \mathrm{S}-\beta_{0}$ thal patients $(32 \%)(\mathrm{p}=0.20)$. V/Q lung scintigraphy was performed at baseline in 47 patients. Lung perfusion was normal in 15 patients (32\%), heterogeneous in $17(36 \%)$ and with mismatched segmental defects in 15 (32\%). The proportion of patients with mismatched segmental perfusion defects was significantly higher in patients with SC SCD compared to patients with SS/S- $\beta_{0}$ thal (85\% versus $9 \%$, $\mathrm{p}<0.001$ ) (figure 2a). CTPA was performed in all patients with abnormal V/Q lung scintigraphy and DSA was performed in 18 patients. Isolated poor subpleural perfusion in the capillary phase of DSA, defined as the absence of perfusion $\leqslant 1.5 \mathrm{~cm}$ from the lateral pleura on the posterior-anterior views and by lateral views of the dorsal area [16], was observed in 14 patients (eight SS/S- $\beta_{0}$ thal and six SC). Lobar, segmental or subsegmental CTEPH was diagnosed in four patients (three SC and one SS) (figure 2b and c).
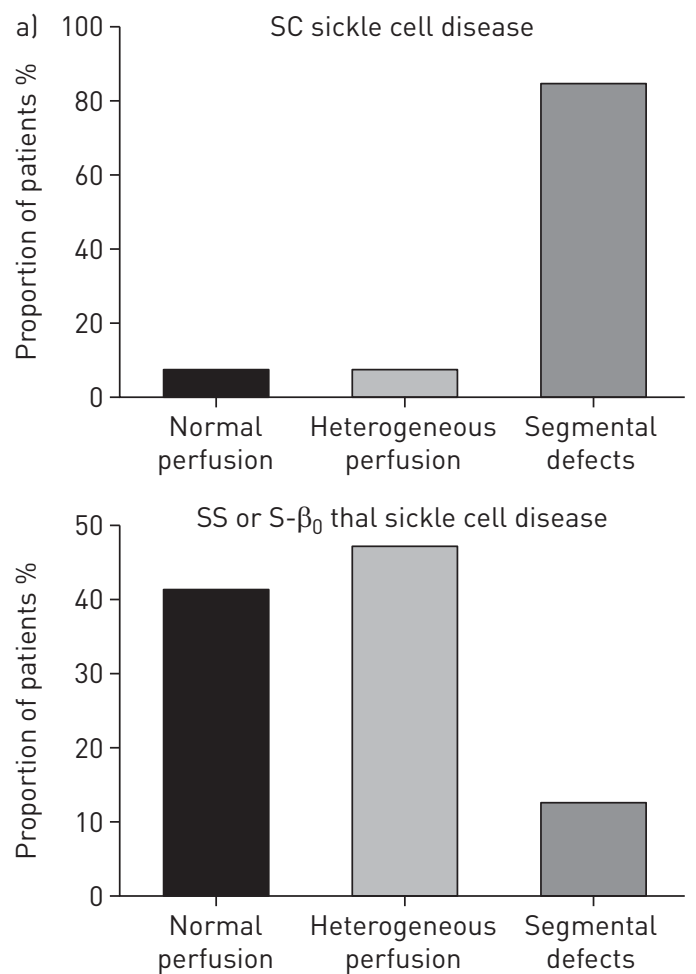

b)

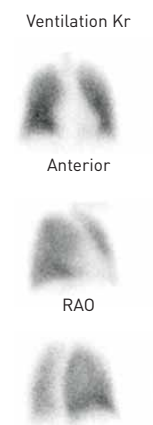

RPO

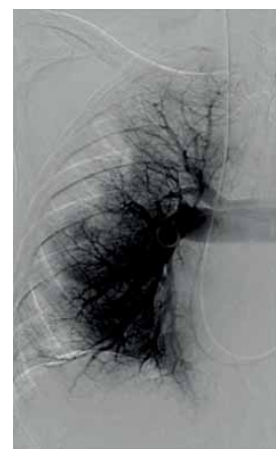

c)

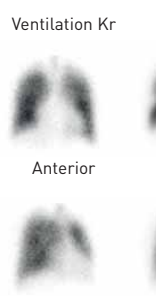

RAO

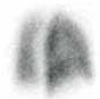

RPO

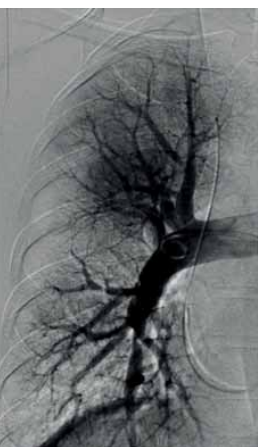

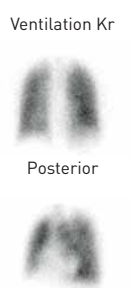

LAO

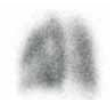

LPO

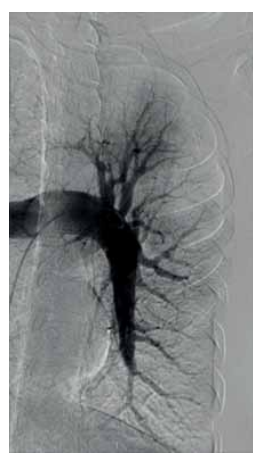

FIGURE 2 Ventilation/perfusion (V/Q) lung scintigraphy and digital subtraction pulmonary angiography (DSA) according to the disease genotype. a) The proportion of patients with normal perfusion, heterogeneous perfusion and segmental perfusion defects on V/Q lung scintigraphy according to the genotype of sickle cell disease. b) A case of homozygous for haemoglobin $S$ (SS) sickle cell disease with heterogeneous perfusion on $V / Q$ lung scintigraphy and diffuse subpleural hypoperfusion on DSA. c) An example of an SC sickle cell disease patient with segmental perfusion defects on V/Q lung scintigraphy and distal chronic thromboembolic pulmonary hypertension on DSA. RAO: right anterior oblique; RPO: right posterior oblique; LAO: left anterior oblique; LPO: left posterior oblique. 
A comparison of characteristics according to the V/Q lung scintigraphy results is reported in table 2. Patients with mismatched segmental perfusion defects more frequently had a SC genotype and had significantly higher haemoglobin levels with significantly lower levels of bilirubin. In contrast, patients with normal V/Q lung scintigraphy had lower levels of haemoglobin with more severe haemolysis. In addition, these patients had more severely impaired 6MWD, as compared to patients with heterogeneous perfusion or mismatched segmental perfusion defects.

\section{Management of precapillary PH}

Medical therapies approved for PAH were prescribed off-label in $23 \mathrm{SS} / \mathrm{S}-\beta_{0}$ thal patients (52\%), at the time of PH diagnosis $(n=14)$ or during follow-up $(n=9)$. Most of them received a monotherapy (14 ERA, one PDE5i and two intravenous epoprostenol). Six patients received initial or sequential combination therapy: ERA and PDE5i $(n=3)$, ERA and intravenous epoprostenol $(n=2)$ and ERA, PDE5i and intravenous prostacyclin $(n=1)$. During the follow-up period, ERA was withdrawn in one patient because of significant elevation of transaminase and in three other patients because of a high cardiac output combined with a postcapillary component induced by treatment. PDE5i was replaced by ERA in one case because of diffuse muscular pain. One SS patient underwent pulmonary endarterectomy surgery. A chronic red blood cell exchange programme was initiated in $22 \mathrm{SS} / \mathrm{S}-\beta_{0}$ thal patients at the time of $\mathrm{PH}$ diagnosis $(n=10)$ or during the follow-up period because of a progression of their pulmonary vascular

\section{TABLE 2 Characteristics according to lung perfusion on ventilation/perfusion scintigraphy}

\begin{tabular}{|c|c|c|c|}
\hline & $\begin{array}{l}\text { Normal } \\
\text { perfusion }\end{array}$ & $\begin{array}{l}\text { Heterogeneous } \\
\text { perfusion }\end{array}$ & $\begin{array}{c}\text { Segmental perfusion } \\
\text { defects }\end{array}$ \\
\hline Number of patients $n$ & 15 & 17 & 15 \\
\hline SC SCD n $(\%)$ & $1(7)$ & $1(6)$ & 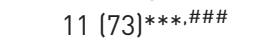 \\
\hline Age years & $43(27-60)$ & $40(18-61)$ & $46(18-63)$ \\
\hline History of pulmonary embolism n (\%) & $4(28)$ & $6(35)$ & $9(60)^{*}$ \\
\hline History of deep vein thrombosis $\mathrm{n}(\%)$ & $1(7)$ & $1(6)$ & $2(13)$ \\
\hline History of ACS $n(\%)$ & $12(80)$ & $10(59)$ & $6(40)$ \\
\hline History of leg ulcers $\mathrm{n}(\%)$ & $5(36)$ & $2(12)$ & $0(0)$ \\
\hline \multicolumn{4}{|l|}{ Treatment regimens at time of $\mathrm{PH}$ diagnosis } \\
\hline Hydroxyurea therapy $\mathrm{n}(\%)$ & $12(80)$ & $5(29)$ & 2 (13) \\
\hline Regular transfusion programme $\mathrm{n}(\%)$ & $3(20)$ & $4(24)$ & $1(7)$ \\
\hline \multicolumn{4}{|l|}{ Biology } \\
\hline Haemoglobin $\mathrm{g} \cdot \mathrm{dL}^{-1}$ & $8.2(6.6-9.6)$ & $9.1(6.5-11.5)$ & $10(7.3-14.2)^{* *}$ \\
\hline Creatinine $\mu \mathrm{mol} \cdot \mathrm{L}^{-1}$ & $81(36-167)$ & $86(57-137)$ & $86(59-128)$ \\
\hline Lactate dehydrogenase IU. $\mathrm{L}^{-1}$ & $1083(413-3034)$ & $1034(334-2691)$ & $665(317-1457)$ \\
\hline Total bilirubin $\mu \mathrm{mol} \cdot \mathrm{L}^{-1}$ & $57(11-223)$ & $69(19-233)$ & $21(8-37)^{* *, \# \#}$ \\
\hline \multicolumn{4}{|l|}{ Arterial gas exchanges } \\
\hline$P_{\mathrm{aO}_{2}} \mathrm{mmHg}$ & $68(43-100)$ & $64(51-71)$ & 74 (59-87) \\
\hline$P_{\mathrm{aO}_{2}} \mathrm{mmHg}$ & $38(27-46)$ & $35(25-45)$ & $38(29-56)$ \\
\hline \multicolumn{4}{|l|}{ Respiratory test } \\
\hline FVC \% & $60(23-107)$ & $72(46-97)$ & $72(44-107)$ \\
\hline $\mathrm{FEV}_{1} \%$ & $54(25-94)$ & $68(45-97)^{*}$ & $69(44-97)^{*}$ \\
\hline TLC \% & $63(36-99)$ & $75(49-97)$ & $73(55-91)$ \\
\hline$D_{\text {LCO }} \%$ & $37(25-66)$ & $52(32-68)^{*}$ & $50(28-81)^{* *}$ \\
\hline$D_{\mathrm{LCO}}$ adjusted for $\mathrm{Hb} \%$ & $45(34-86)$ & $62(37-81)^{*}$ & $56(37-98)^{*}$ \\
\hline 6-min walk distance $\mathrm{m}$ & $246(0-501)$ & $366(167-569)$ & $369(0-554)$ \\
\hline \multicolumn{4}{|l|}{ Haemodynamics } \\
\hline RAP $\mathrm{mmHg}$ & $8(3-18)$ & $9(0-34)$ & $7(0-11)$ \\
\hline mPAP $\mathrm{mmHg}$ & $37(27-54)$ & $37(25-62)$ & $38(27-54)$ \\
\hline PAWP $\mathrm{mmHg}$ & $10(3-14)$ & $7(1-12)^{*}$ & $9(1-15)$ \\
\hline Cardiac index $\mathrm{L} \cdot \mathrm{min}^{-1} \cdot \mathrm{m}^{-2}$ & $4.8(2.4-7.5)$ & $3.8(1.8-5.6)$ & $3.3(2.1-4.9)^{* *}$ \\
\hline PVR Wood units & $4.3(1.7-21)$ & $5.9(1.9-20.7)$ & $5.4(1.7-10.7)^{*}$ \\
\hline Mixed oxygen venous saturation \% & $60(32-72)$ & $63(47-80)$ & $61(44-86)$ \\
\hline \multicolumn{4}{|c|}{ 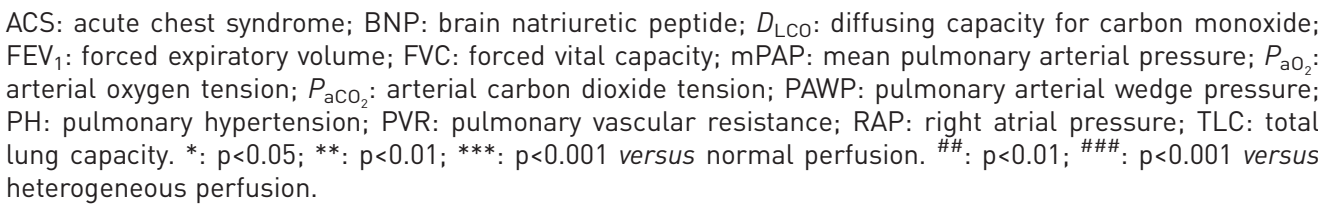 } \\
\hline
\end{tabular}


disease $(n=12)$. In comparison with patients not treated by exchange transfusions, those treated with exchange transfusions were more frequently in NYHA-FC III or IV ( $82 \%$ versus $50 \%, \mathrm{p}=0.026)$ and had significantly higher median levels of right atrial pressure (8 versus $5 \mathrm{mmHg}, \mathrm{p}=0.005)$ and $\mathrm{mPAP}$ (36 versus $30 \mathrm{mmHg}, \mathrm{p}=0.017)$.

11 SC patients had segmental perfusion defects. All of them were treated with long-term anticoagulation. Eight were treated with medical therapies including monotherapy with ERA $(n=5)$, monotherapy with PDE5i $(n=1)$ and combination therapy with guanylate cyclase stimulator riociguat and ERA $(n=2)$. Three SC patients were treated with balloon pulmonary angioplasty in combination with PAH-targeted therapy in one. A red blood cell exchange programme was initiated in one SC patient.

RHC was repeated after a median time of 9.6 months in 16 patients who did not benefit from surgical, interventional or medical therapy for PH and had no change in SCD management as first line therapy. In this group of patients, we observed a non-significant change in median PVR after PH diagnosis from 2.7 WU to $3.5 \mathrm{WU}(\mathrm{p}=0.09)$. In contrast, patients who received medical therapies approved for $\mathrm{PAH}$ and/ or SCD management had a decrease in median PVR from 4.6 to $3.3 \mathrm{WU}(\mathrm{p}=0.0014)$ after a median follow-up of 6.6 months. Individual data of PVR at baseline and first evaluation are illustrated in supplementary figure $\mathrm{S} 1$.

\section{Survival and baseline prognostic factors}

Over a median (interquartile range) follow-up period of 38 (11-56) months, 21 patients (36\%) died. Overall survival rates were $91 \%, 80 \%$ and $60 \%$ at 1,3 and 5 years, respectively. Causes of death were recorded in 14 out of 21 cases (67\%). Among them, 13 patients died from right heart failure and one from sudden death. The results of univariate analysis of baseline variables and survival are shown in table 3 . Mortality was not associated with the sex of the patient or any haemodynamic variables. Only NYHA-FC III or IV was associated with worse survival. The prognosis of the SS/S- $\beta_{0}$ thal and SC groups was similar. Baseline 6MWD and PVR did not influence survival (figure 3).

\section{Discussion}

We report here the baseline clinical characteristics and long-term outcomes of a population of SCD patients with precapillary PH diagnosed by RHC (after screening with transthoracic Doppler echocardiography). Our results highlight the wide spectrum of phenotypes observed in this population, and the influence of the SCD genotype on clinical and functional characteristics. Thromboembolic mechanisms seem to play a major role in the occurrence of precapillary $\mathrm{PH}$ in SCD, especially in the setting of a SC genotype. Indeed, SC patients presented with a history of acute venous thromboembolism

\section{TABLE 3 Prognostic factors (univariate analysis)}

\begin{tabular}{|c|c|c|}
\hline & Hazard ratio $(95 \% \mathrm{CI})$ & p-value \\
\hline Age years & $1.03(0.98-1.08)$ & 0.2 \\
\hline Female & $1.85(0.74-4.59)$ & 0.5 \\
\hline BMI $\mathrm{kg} \cdot \mathrm{m}^{-2}$ & $1.1(0.98-1.23)$ & 0.09 \\
\hline SC genotype & $0.89(0.25-3.13)$ & 0.9 \\
\hline NYHA-FC I or II & $0.19(0.05-0.7)$ & 0.01 \\
\hline $6 M W D>360 \mathrm{~m}$ & $0.63(0.26-2.8)$ & 0.3 \\
\hline Abnormal V/Q lung scintigraphy & $0.4(0.13-1.21)$ & 0.1 \\
\hline \multicolumn{3}{|l|}{ Haemodynamics } \\
\hline RAP mmHg & $0.96(0.88-1.04)$ & 0.3 \\
\hline mPAP mmHg & $1(0.95-1.04)$ & 0.9 \\
\hline PAWP $\mathrm{mmHg}$ & $0.98(0.87-1.12)$ & 0.8 \\
\hline Cardiac index $\mathrm{L} \cdot \mathrm{min}^{-1} \cdot \mathrm{m}^{-2}$ & $0.93(0.68-1.27)$ & 0.6 \\
\hline PVR Wood units & $0.99(0.92-1.08)$ & 0.9 \\
\hline \multicolumn{3}{|l|}{ Biology } \\
\hline Haemoglobin $\mathrm{g} \cdot \mathrm{dL}^{-1}$ & $0.92(0.67-1.25)$ & 0.6 \\
\hline Lactate dehydrogenase IU. $\mathrm{L}^{-1}$ & $1(1-1.001)$ & 0.4 \\
\hline Creatinine $\mu \mathrm{mol} \cdot \mathrm{L}^{-1}$ & $1.01(0.99-1.03)$ & 0.2 \\
\hline High BNP or NT-proBNP & $1.3(0.46-3.65)$ & 0.6 \\
\hline
\end{tabular}

BNP: brain natriuretic peptide; PAWP: pulmonary arterial wedge pressure; mPAP: mean pulmonary arterial pressure; NT-proBNP: N-terminal pro-brain natriuretic peptide; NYHA-FC: New York Heart Association functional class; PH: pulmonary hypertension; PVR: pulmonary vascular resistance; RAP: right atrial pressure; V/Q: ventilation/perfusion. 

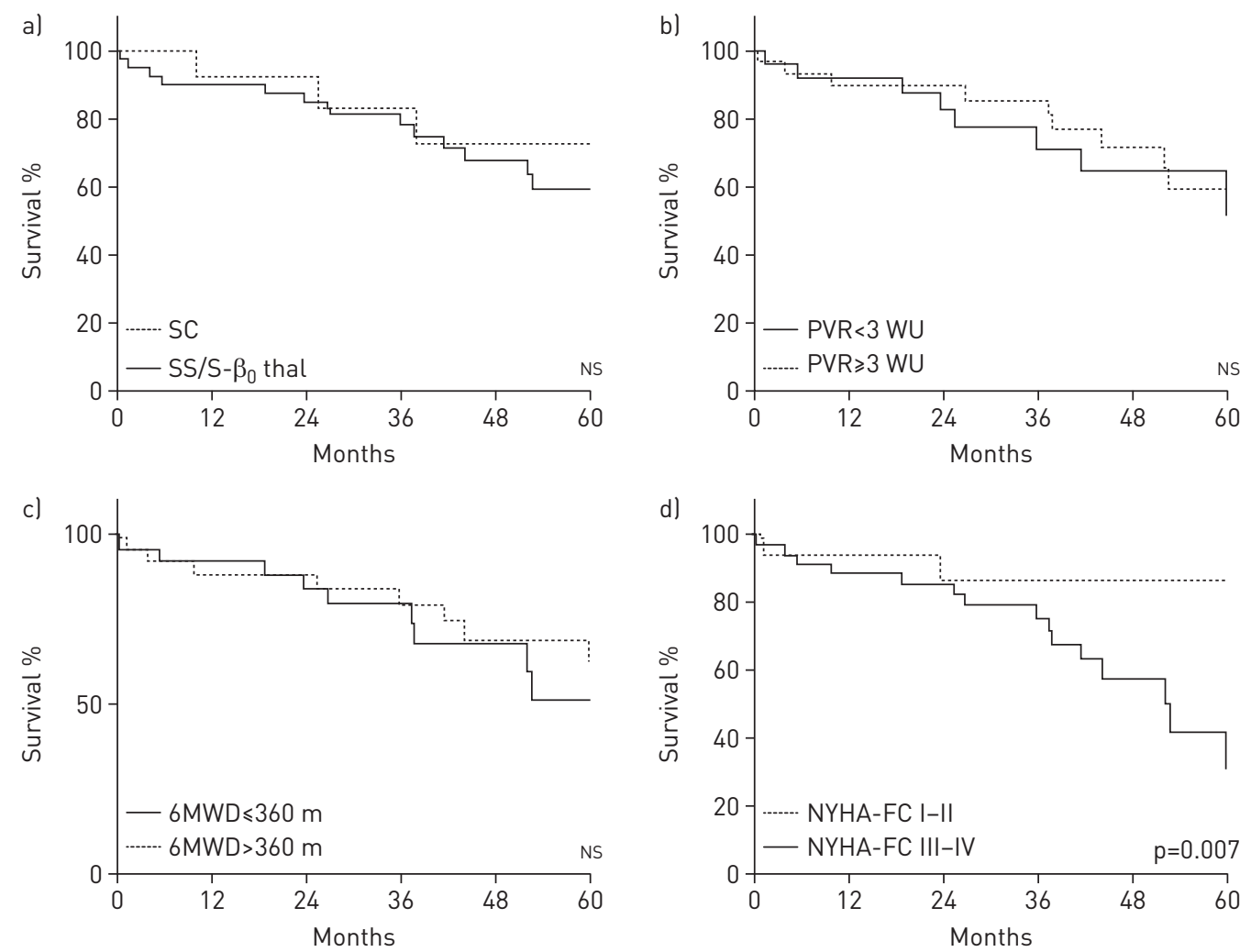

FIGURE 3 Overall survival according to a) sickle cell disease genotype, b) pulmonary vascular resistance (PVR), c) 6-min walk distance (6MWD) and d) New York Heart Association functional class (NYHA-FC). NS: not statistically significant.

in more than $50 \%$ of cases and V/Q lung scintigraphy showed mismatched segmental perfusion defects in more than $80 \%$. In $S S / S-\beta_{0}$ thal patients, heterogeneous lung perfusion associated with poor subpleural perfusion on DSA was observed in half of the cases. The functional status of these patients is influenced by their underlying genotype and the presence of a thromboembolic component, with higher 6MWD reported in SC patients and in those with a diagnosis of CTEPH. By contrast, the SCD genotype and haemodynamics did not influence prognosis. Only NYHA-FC III or IV at diagnosis was associated with worse survival.

Precapillary PH appears as a late complication of SCD with a median age of 43 years at the time of diagnosis in our study, irrespective of the SCD genotype. This is in agreement with previous screening studies performed in SCD $[2-4,17]$. Exercise capacity and haemodynamics were influenced by SCD genotype with a significantly higher 6MWD observed in SC patients, while cardiac index was lower and PVR levels were higher. We can stipulate that haemodynamic variables are not only influenced by the severity of pulmonary vascular disease but also by rheologic aspects of anaemia in addition to the viscosity differences between SS/S- $\beta_{0}$ thal and SC patients. While SS SCD and S- $\beta_{0}$ thal are associated with more severe haemolysis and anaemia, haemoglobin SC disease is characterised by higher haemoglobin levels leading to hyperviscosity. According to the Hagen-Poiseuille equation, PVR is dependent on fluid viscosity, which can be influenced by several variables such as haematocrit, genotype and severity of haemolysis in SCD $[18,19]$. The exercise capacity of SS/S- $\beta_{0}$ thal patients is also influenced by factors other than $\mathrm{PH}$ itself, as these patients present with lower levels of haemoglobin and more restrictive lung disease. Previous studies have reported a high rate of dyspnoea in SS SCD, due to multiple causes including anaemia, musculoskeletal disorders, cardiopulmonary complications and others [20].

Our study supports the contributory role of thromboembolic mechanisms in precapillary $\mathrm{PH}$ and, more frequently, in those with the SC genotype. SCD is associated with a chronic hypercoagulable state due to multiple mechanisms including chronic haemolysis itself, inflammation, asplenism, oxidative stress or intrinsic coagulation activation [21]. This is associated with a higher risk of acute venous thromboembolic events [22]. We observed in our study a high prevalence of former acute venous thromboembolic events in SCD patients with precapillary $\mathrm{PH}$, reaching $30 \%$ in the $S S / S-\beta_{0}$ thal genotype and $50 \%$ in the SC 
genotype. The low prevalence of deep vein thrombosis compared to the high prevalence of pulmonary artery thrombosis could suggest a key role played by local thrombotic processes. Similarly, MeкоNTSO DESSAP et al. [23] observed a prevalence of pulmonary artery thrombosis of $17 \%$ in acute chest syndrome, while all patients were free of proximal deep venous thrombosis. Previous autopsy studies reported in situ thrombosis in small pulmonary vessels in a high proportion of patients [5]. In our cohort, V/Q lung scintigraphy was frequently abnormal in this population as previously described [17], with a high frequency of mismatched segmental perfusion defects in SC patients. Similarly, a recent study observed that perfusion abnormalities on V/Q scintigraphy were associated with a higher proportion of $\mathrm{PH}$ [24]. In our present report, we have performed DSA in 18 patients, making it possible to identify two main patterns: patients with isolated poor subpleural perfusion and patients with lobar, segmental or subsegmental CTEPH lesions, as illustrated in figure 2. Interestingly, there was a predominance of male patients in the SC population. This is consistent with previous studies that showed a lower risk of viscosity-related morbidity in female patients due to a lower Hb level linked to menstrual blood losses [25].

These observations have important implications for the management of SCD patients with precapillary $\mathrm{PH}$. Screening for thromboembolic disease by V/Q scintigraphy is a mandatory initial step in the care of SCD patients with PH. Indeed, a diagnosis of CTEPH yields specific management options including pulmonary endarterectomy for operable patients with proximal lesions or medical therapies with or without balloon pulmonary angioplasty for inoperable patients. Several cases of successful pulmonary endarterectomy have been reported in SCD patients [9-12]. In our study, three SC patients with inoperable CTEPH were treated with balloon pulmonary angioplasty [26, 27], leading to $29 \%$ and $43 \%$ decreases in PVR in two patients, and normalisation of mPAP at rest in the third one (data not shown). Moreover, the high proportion of lung perfusion segmental defects in SC patients supports the use of anticoagulation to reduce the risk of thromboembolic disease and of phlebotomy to decrease viscosity-related morbidity in those patients.

V/Q lung scintigraphy was normal in one third of SCD patients with precapillary PH and included a vast majority of patients with SS/S- $\beta_{0}$ thal, who have a pronounced haemolytic phenotype (lower haemoglobin levels, higher haemolytic biomarkers and higher frequency of ulcers). Despite lower levels of PVR in this subpopulation, these patients had severe exercise limitation. Chronic blood cell exchange programmes or hydroxyurea could be interesting options in this subpopulation [28].

Our study confirms the poor prognosis of precapillary PH complicating SCD with a survival rate of $70 \%$ at 3 years. It also illustrates the difficulty in identifying robust prognostic factors in this population. Paradoxically, the SC genotype was not associated with a better prognosis in the present study. However, because this genotype is frequently associated with CTEPH, the impact of recent therapeutic advances, such as balloon pulmonary angioplasty, will have to be analysed in the future. Recently, a case series study reported a beneficial effect of riociguat in six patients with SCD and PH related to chronic thromboembolic disease [29]. One important piece of information is that haemodynamic parameters such as PVR did not influence outcomes. In a previous study, pulmonary pressures and PVR were identified as haemodynamic predictors of mortality in a cohort of adults with SCD. In contrast with our study, patients with and without $\mathrm{PH}$ were included, highlighting the prognostic impact of PH related to SCD [4]. Only NYHA-FC I and II at time of diagnosis was associated with a better prognosis, but it was present in only one third of patients. This observation illustrates the potential interest of PH screening in SCD.

Specific clinical trials are lacking or have failed to demonstrate a positive effect of medical therapies approved for $\mathrm{PAH}$ in precapillary $\mathrm{PH}$ due to SCD. In addition, the reinforcement of SCD management with hydroxyurea and/or chronic transfusion may be an interesting management approach but no prospective evaluation has been performed to test these therapeutic approaches on outcomes of patients with precapillary PH due to SCD [28]. Despite the lack of evidence, $50 \%$ of our patients were treated with medical therapies approved for $\mathrm{PAH}$ and a red blood cell exchange programme was initiated after the diagnosis of $\mathrm{PH}$ in one third of them. Our study did not find any impact of these approaches on long-term survival, but its retrospective nature and the small number of patients are not adequate to detect a significant effect. Because of the dramatic impact of $\mathrm{PH}$ on the functional status and vital prognosis of SCD patients, multicentre prospective studies are warranted. Interestingly, we observed that patients who did not receive medical therapies approved for $\mathrm{PAH}$ and/or reinforcement of SCD treatment with red blood cell exchanges or hydroxyurea as first line therapies displayed a progressive haemodynamic compromise (increased PVR at follow-up).

Our results have limitations inherent to retrospective studies and relatively limited numbers of patients. Due to the nature of our registry being mainly focused on $\mathrm{PH}$, available data on acute complications of SCD during the follow-up period and their potential impact on survival are lacking. However, cause of death was due to $\mathrm{PH}$ in all cases, when identified. Moreover, SCD patients with precapillary $\mathrm{PH}$ were 
evaluated over a long period of time (from 2000 to 2017), and the initial management of PH was influenced by the available tools and growing expertise. Also, it is difficult to interpret the respective effects of medical therapies approved for PAH and of hydroxyurea and red blood cell exchange programmes, which are often used together initially or sequentially in our management strategy as recommended by the 2014 American Thoracic Society clinical practice guideline for the management of $\mathrm{PH}$ of SCD. This gives 1) a weak recommendation for medical therapies approved for PAH for select patients with SCD with RHC-confirmed pre-capillary $\mathrm{PH}$ with elevated PVR, and 2) a strong recommendation for hydroxyurea as a first line therapy and a weak recommendation for chronic transfusions as an alternative therapy [8]. We believe that our observations will enrich the literature and may help guide the future development of novel treatment strategies, which are urgently needed.

In conclusion, our series shows that precapillary PH complicating SCD is a severe and complex condition with an important impact on survival, irrespective of SCD genotype. Thromboembolic mechanisms contribute to a large proportion of SCD patients with precapillary $\mathrm{PH}$, especially in the SC genotype. This finding supports systematic CTEPH screening with V/Q lung scintigraphy in SCD patients with precapillary $\mathrm{PH}$. The high prevalence of precapillary $\mathrm{PH}$ in SCD, its impact on outcomes and the association between NYHA-FC at diagnosis and prognosis support $\mathrm{PH}$ screening programmes by transthoracic Doppler echocardiography in SCD. Finally, management with medical therapies approved for PAH and reinforcement of SCD management may have a positive impact on haemodynamics, but further studies are needed to update the guidelines for treating precapillary $\mathrm{PH}$ complicating SCD [8]. The clinical heterogeneity of SCD patients with precapillary PH underscores the importance of a careful aetiological assessment for each patient, managed by a multidisciplinary team involving PH and SCD experts.

Acknowledgements: We wish to acknowledge Laurence Rottat for her help in obtaining the data for this study and her hard work in managing data in the French PAH Registry.

Author contributions: L. Savale, M. Humbert and P. Bartolucci were responsible for design, analysis and interpretation of data. L. Savale, A. Habibi and P. Bartolucci participated in data collection. L. Savale, A. Habibi, F. Lionnet and M. Humbert prepared the manuscript. All authors were responsible for and contributed to manuscript review and revision. All authors approved the final version of the manuscript.

Conflict of interest: L. Savale reports grants, personal fees and non-financial support from Actelion, MSD, GSK and Bayer, outside the submitted work. A. Habibi has nothing to disclose. F. Lionnet has nothing to disclose. B. Maitre has nothing to disclose. V. Cottin reports personal fees for consultancy and lectures, and non-financial support for travel to meetings from Actelion, grants, personal fees for consultancy and lectures, and non-financial support for travel to meetings from Boehringer Ingelheim and Roche, personal fees for consultancy from Bayer/MSD and Galapagos, personal fees for adjudication committee work from Gilead, personal fees for consultancy and lectures from Novartis and Astra Zeneca, grants from Sanofi, and personal fees for data monitoring committee work from Promedior and Celgene, outside the submitted work. X. Jais reports grants, personal fees and non-financial support from Actelion, MSD, GSK and Bayer, outside the submitted work. A. Chaouat has nothing to disclose. E. Artaud-Macari has nothing to disclose. M. Canuet reports personal fees for advisory board work from Actelion, non-financial support for travel to meetings from France Oxygene, outside the submitted work. G. Prevot has nothing to disclose. C. Chantalat-Auger has nothing to disclose. D. Montani reports grants and personal fees from Actelion and Bayer, personal fees from GSK, MSD and Pfizer, outside the submitted work. O. Sitbon reports grants, personal fees and non-financial support from Actelion Pharmaceuticals and GlaxoSmithKline, grants and personal fees from Bayer HealthCare, grants and non-financial support from Merck, personal fees from Arena Pharmaceuticals, outside the submitted work. F. Galacteros has nothing to disclose. G. Simonneau reports grants, personal fees and non-financial support from Actelion Pharmaceuticals and GlaxoSmithKline, grants and personal fees from Bayer HealthCare, grants and non-financial support from Merck, personal fees from Arena Pharmaceuticals, outside the submitted work. F. Parent has nothing to disclose. P. Bartolucci has nothing to disclose. M. Humbert has relationships with drug companies including Actelion, Bayer, GSK, Novartis, Pfizer and United Therapeutics. In addition to being investigator in trials involving these companies, relationships include consultancy service and membership of scientific advisory boards.

\section{References}

1 Gladwin MT. Cardiovascular complications and risk of death in sickle-cell disease. Lancet Lond Engl 2016; 387: 2565-2574.

2 Parent F, Bachir D, Inamo J, et al. A hemodynamic study of pulmonary hypertension in sickle cell disease. $N$ Engl J Med 2011; 365: 44-53.

3 Fonseca GHH, Souza R, Salemi VMC, et al. Pulmonary hypertension diagnosed by right heart catheterisation in sickle cell disease. Eur Respir J 2012; 39: 112-118.

4 Mehari A, Alam S, Tian X, et al. Hemodynamic predictors of mortality in adults with sickle cell disease. Am J Respir Crit Care Med 2013; 187: 840-847.

5 Adedeji MO, Cespedes J, Allen K, et al. Pulmonary thrombotic arteriopathy in patients with sickle cell disease. Arch Pathol Lab Med 2001; 125: 1436-1441.

6 Gordeuk VR, Castro OL, Machado RF. Pathophysiology and treatment of pulmonary hypertension in sickle cell disease. Blood 2016; 127: 820-828.

7 Gladwin MT, Sachdev V, Jison ML, et al. Pulmonary hypertension as a risk factor for death in patients with sickle cell disease. N Engl J Med 2004; 350: 886-895. 

diagnosis, risk stratification, and management of pulmonary hypertension of sickle cell disease. Am J Respir Crit Care Med 2014; 189: 727-740.

9 Mahesh B, Besser M, Ravaglioli A, et al. Pulmonary endarterectomy is effective and safe in patients with haemoglobinopathies and abnormal red blood cells: the Papworth experience. Eur J Cardiothorac Surg 2016; 50: 537-541.

10 Freeman AT, Ataga KI. Pulmonary endarterectomy as treatment for chronic thromboembolic pulmonary hypertension in sickle cell disease. Am J Hematol 2015; 90: E223-E224.

11 Marques MB, Wille KM, Ren Z, et al. Successful pulmonary thromboendarterectomy in a patient with sickle cell disease treated with a single preoperative red blood cell exchange. Transfusion (Paris) 2014; 54: 1901-1902.

12 Jerath A, Murphy P, Madonik M, et al. Pulmonary endarterectomy in sickle cell haemoglobin C disease. Eur Respir J 2011; 38: 735-737.

13 Kim NH, Delcroix M, Jais X, et al. Chronic thromboembolic pulmonary hypertension. Eur Respir J 2019; 53: 1801915.

14 Galiè N, Humbert M, Vachiery J-L, et al. 2015 ESC/ERS Guidelines for the diagnosis and treatment of pulmonary hypertension: The Joint Task Force for the Diagnosis and Treatment of Pulmonary Hypertension of the European Society of Cardiology (ESC) and the European Respiratory Society (ERS). Eur Heart J 2016; 37: 67-119.

15 Galiè N, Humbert M, Vachiery J-L, et al. 2015 ESC/ERS Guidelines for the diagnosis and treatment of pulmonary hypertension. Eur Respir J 2015; 46: 903-975.

16 Taniguchi $\mathrm{Y}$, Brenot $\mathrm{P}$, Jais X, et al. Poor subpleural perfusion predicts failure after balloon pulmonary angioplasty for nonoperable chronic thromboembolic pulmonary hypertension. Chest 2018; 154: 521-531.

17 Anthi A, Machado RF, Jison ML, et al. Hemodynamic and functional assessment of patients with sickle cell disease and pulmonary hypertension. Am J Respir Crit Care Med 2007; 175: 1272-1279.

18 Connes P, Alexy T, Detterich J, et al. The role of blood rheology in sickle cell disease. Blood Rev 2016; 30: $111-118$.

19 Vanderpool RR, Naeije R. Hematocrit-corrected pulmonary vascular resistance. Am J Respir Crit Care Med 2018; 198: 305-309.

20 Delclaux C, Zerah-Lancner F, Bachir D, et al. Factors associated with dyspnea in adult patients with sickle cell disease. Chest 2005; 128: 3336-3344.

21 Sparkenbaugh E, Pawlinski R. Prothrombotic aspects of sickle cell disease. J Thromb Haemost 2017; 15: 1307-1316.

22 Brunson A, Lei A, Rosenberg AS, et al. Increased incidence of VTE in sickle cell disease patients: risk factors, recurrence and impact on mortality. Br J Haematol 2017; 178: 319-326.

23 Mekontso Dessap A, Deux J-F, Abidi N, et al. Pulmonary artery thrombosis during acute chest syndrome in sickle cell disease. Am J Respir Crit Care Med 2011; 184: 1022-1029.

24 Mehari A, Igbineweka N, Allen D, et al. Abnormal ventilation-perfusion scan is associated with pulmonary hypertension in sickle cell adults. J Nucl Med 2019; 60: 86-92.

25 Lionnet F, Hammoudi N, Stojanovic KS, et al. Hemoglobin sickle cell disease complications: a clinical study of 179 cases. Haematologica 2012; 97: 1136-1141.

26 Madani M, Ogo T, Simonneau G. The changing landscape of chronic thromboembolic pulmonary hypertension management. Eur Respir Rev 2017; 26: 170105.

27 Lang I, Meyer BC, Ogo T, et al. Balloon pulmonary angioplasty in chronic thromboembolic pulmonary hypertension. Eur Respir Rev 2017; 26: 160119.

28 Turpin M, Chantalat-Auger C, Parent F, et al. Chronic blood exchange transfusions in the management of pre-capillary pulmonary hypertension complicating sickle cell disease. Eur Respir J 2018; 52: 1800272.

29 Weir NA, Conrey A, Lewis D, et al. Riociguat use in sickle cell related chronic thromboembolic pulmonary hypertension: a case series. Pulm Circ 2018; 8: 2045894018791802. 